University of South Carolina

Scholar Commons

$10-31-2005$

\title{
Terahertz Studies of the Dielectric Response and Second-Order Phonons in a GaSe Crystal
}

\author{
B. L. Yu \\ F. Zeng \\ V. Kartazayev \\ R. R. Alfano \\ K. C. Mandal \\ University of South Carolina - Columbia, mandalk@engr.sc.edu
}

Follow this and additional works at: https://scholarcommons.sc.edu/elct_facpub

Part of the Electrical and Electronics Commons, and the Optics Commons

\author{
Publication Info \\ Published in Applied Physics Letters, Volume 87, Issue 18, 2005, pages 182104-1-182104-3. \\ (c) Applied Physics Letters 2005, American Institute of Physics \\ Yu, B. L., Zeng, F., Kartazayev, V., Alfano, R. R., \& Mandal, K. C. (31 October 2005). Terahertz studies of the \\ dielectric response and second-order phonons in a GaSe crystal. Applied Physics Letters, 87(18), \\ \#182104. \\ http://dx.doi.org/10.1063/1.2093944 \\ http://scitation.aip.org/content/aip/journal/apl/87/18/10.1063/1.2093944
}

This Article is brought to you by the Electrical Engineering, Department of at Scholar Commons. It has been accepted for inclusion in Faculty Publications by an authorized administrator of Scholar Commons. For more information, please contact digres@mailbox.sc.edu. 


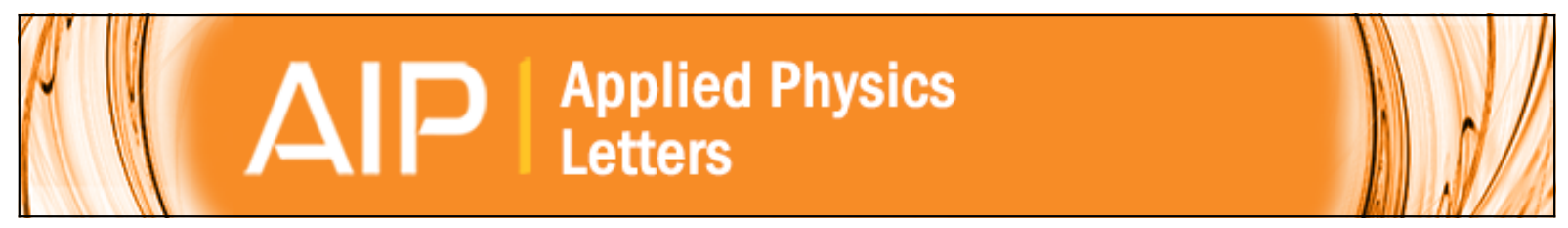

Terahertz studies of the dielectric response and second-order phonons in a GaSe crystal

B. L. Yu, F. Zeng, V. Kartazayev, R. R. Alfano, and Krishna C. Mandal

Citation: Applied Physics Letters 87, 182104 (2005); doi: 10.1063/1.2093944

View online: http://dx.doi.org/10.1063/1.2093944

View Table of Contents: http://scitation.aip.org/content/aip/journal/apl/87/18?ver=pdfcov

Published by the AIP Publishing

\section{Articles you may be interested in}

Response to "Comment on 'GaSe1-xSx and GaSe1-xTex thick crystals for broadband terahertz pulses generation'” [Appl. Phys. Lett. 100, 136103 (2012)]

Appl. Phys. Lett. 100, 136104 (2012); 10.1063/1.3698458

GaSe1-xSx and GaSe1-xTex thick crystals for broadband terahertz pulses generation

Appl. Phys. Lett. 99, 081105 (2011); 10.1063/1.3617438

Erratum: "Terahertz studies of the dielectric response and second-order phonons in a GaSe crystal" [Appl. Phys. Lett.87, 182104 (2005)]

Appl. Phys. Lett. 88, 159902 (2006); 10.1063/1.2194647

Terahertz dielectric properties of high-resistivity single-crystal $\mathrm{ZnO}$

Appl. Phys. Lett. 88, 021103 (2006); 10.1063/1.2164903

GaSe crystals for broadband terahertz wave detection

Appl. Phys. Lett. 85, 863 (2004); 10.1063/1.1779959

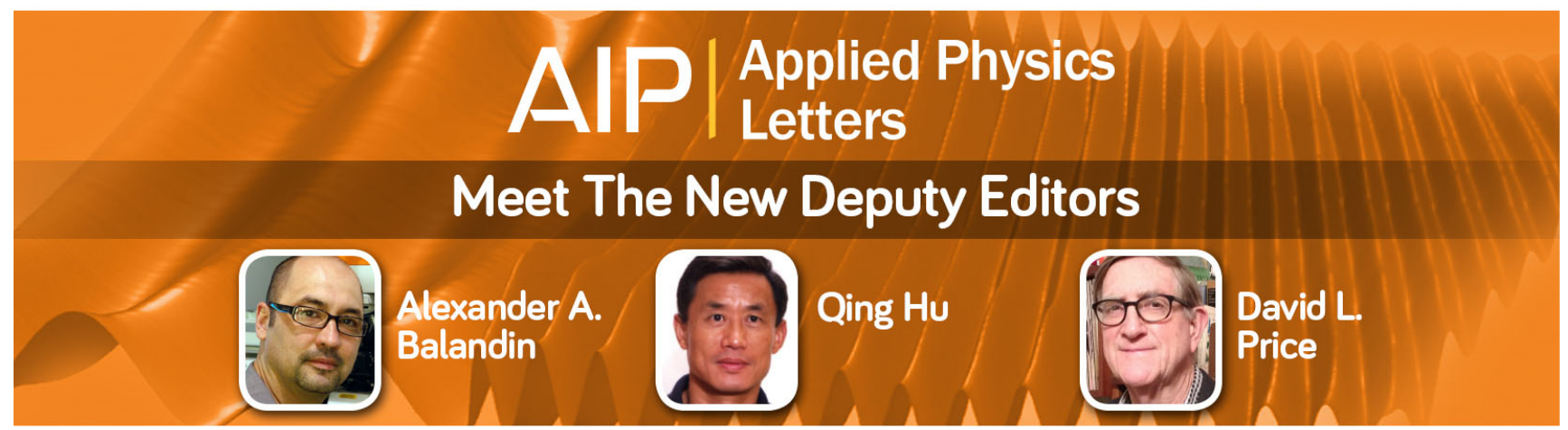




\title{
Terahertz studies of the dielectric response and second-order phonons in a GaSe crystal
}

\author{
B. L. Yu, F. Zeng, V. Kartazayev, and R. R. Alfano ${ }^{a)}$ \\ Institute for Ultrafast Spectroscopy and Lasers and New York State Center for Advanced Technology \\ for Ultrafast Photonics Materials and Applications, Physics Department, The City College \\ of the City University of New York, New York, New York 10031 \\ Krishna C. Mandal \\ EIC Laboratories, Inc., 111 Downey Street, Norwood, Massachusetts 02062
}

(Received 15 June 2005; accepted 17 August 2005; published online 25 October 2005)

\begin{abstract}
The dielectric function and momentum relaxation time of carriers for a single-crystal GaSe were investigated using terahertz time-domain spectroscopy over the frequency range from 0.4 to $2.4 \mathrm{THz}$. The key parameters determined from $\mathrm{THz}$ data using the Drude model are: the plasma frequency $\omega_{p}=2.6 \pm 0.2 \mathrm{THz}$, the average momentum relaxation time $\langle\tau\rangle=56 \pm 2 \mathrm{fs}$, and the mobility $\mu=89 \mathrm{~cm}^{2} / \mathrm{V}$ s for electrons. The THz absorption spectrum showed resonance structures attributed to the difference frequency combinations associated with acoustical and optical phonons. (C) 2005 American Institute of Physics. [DOI: 10.1063/1.2093944]
\end{abstract}

Gallium selenide ( $\mathrm{GaSe}$ ) is a promising semiconductor crystal for nonlinear optics and $\mathrm{THz}$ generation with an extremely large bandwidth up to $41 \mathrm{THz}$. ${ }^{1,2} \mathrm{GaSe}$ is a negative uniaxial layered semiconductor with hexagonal structure of $\overline{62} \mathrm{~m}$ point group with a direct band gap $E_{g}^{d}=2.202 \mathrm{eV}$ and an indirect gap $E_{g}^{i}=1.995 \mathrm{eV}$ at $T=300 \mathrm{~K}$. The longitudinal and transverse optical phonons are located at 254 and $213 \mathrm{~cm}^{-1}$, respectively. ${ }^{3,4}$ Recently, GaSe was used in the generation of 0.18-5.27 THz electromagnetic waves using a differencefrequency technique ${ }^{4}$ and also used in phase-matched optical rectification with a Ti:sapphire laser from 0.4 to $41 \mathrm{THz}{ }^{1}$ GaSe is becoming a material of choice for a $\mathrm{THz}$ emitter and sensor. Time-domain spectroscopy (THz-TDS) can achieve a high signal-to-noise ratio $\left(\sim 10^{4}\right)$ using coherent phase detection for characterizing a variety of materials, including molecular vapors, liquids, semiconductors, superconductors, biomedical molecules and tissues, nanostructures materials, and artificial metallic structures. ${ }^{5-7}$ Recently, THz-TDS has also been used to investigate the lattice absorption associated with the fundamental and second order phonon resonances in semiconductors. ${ }^{8}$

This letter reports on the dielectric response measurements in the far-infrared absorption of $\mathrm{z}$-cut crystals of doped GaSe using THz-TDS in the frequency range $0.4-2.4 \mathrm{THz}$. Absorption peaks at $0.80 \mathrm{THz}$ $\left(26.7 \mathrm{~cm}^{-1}\right), 1.24 \mathrm{THz}\left(41.3 \mathrm{~cm}^{-1}\right), 1.57 \mathrm{THz}\left(52.3 \mathrm{~cm}^{-1}\right)$, and $1.84 \mathrm{THz}\left(61.3 \mathrm{~cm}^{-1}\right)$ were observed which are attributed to the difference frequency combinations of acoustical and optical phonons (see Table I). The key parameters extracted from data analysis using the Drude model for the dielectric response are the plasma frequency $\omega_{p}$, the average momentum relaxation time $\langle\tau\rangle$, the mobility and the frequencies of the combinations of acoustical and optical phonons in the $\mathrm{THz}$ region.

$\mathrm{THz}$ pulses were generated using 70 fs optical pulses by optical rectification in a $\mathrm{ZnTe}$ crystal. The details of the experimental system is described in Refs. 7 and 9. The entire setup was placed in an airtight enclosure purged with dry

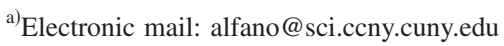

nitrogen. Sample of z-cut GaSe crystals are unintentionally doped single crystal with thicknesses of $\sim 30 \mu \mathrm{m}, \sim 80 \mu \mathrm{m}$, and $140 \mu \mathrm{m}$, free standing crystalline plates. It was grown at the EIC Lab. All measurements were performed at room temperature $(296 \mathrm{~K})$. The signal-noise-ratio is $10^{4}$ for the measurement system.

The time profiles of the $\mathrm{THz}$ pulse transmitted through the samples and without a sample are shown in Fig. 1. From these temporal profiles, the delay due to the sample index and thickness is obtained as $\Delta t=1.16 \pm 0.02$ ps for $140 \mu \mathrm{m}$. Using the measurement of the time delay $\Delta t$, the group index of refraction is calculated as $n_{g}=1+c \Delta t / d=3.3$, where the $d(=140 \mu \mathrm{m})$ is the thickness of the sample, and $c$ is the speed of light in vacuum. The result for $n_{g}$ is consistent with the measured values in the region of $0.4-2.4 \mathrm{THz}$ at room temperature [see Fig. 2(b)]. In this study, the normal incident $\mathrm{THz}$ beam had horizontal polarization which corresponds to $\theta=0$ for a $\mathrm{z}$-cut crystal, where $\theta$ is the angle between the propagation direction of the incident beam and the optical axis (normal to the surface). In this geometry, the refractive index measured is the ordinary index, $n_{0}$.

Because of the relatively clear separation in the time domain between the main transmitted pulse and the first internal reflection, the initial data analysis was performed on the main pulse. For this simple case the frequency spectrum of only the first transmitted pulse shows no oscillation. The maximum recorded delay was limited to 4.9 ps by the translation stage. The refractive index at different frequencies $(\nu)$ is determined by the phase difference as $n(\nu)=1$ $+c \Delta \phi / 2 \pi \nu d$, and the absorption coefficient is calculated as $\alpha(\nu)=-(1 / d) \ln [\eta \cdot P s(\nu, d) / \operatorname{Pr}(\nu, d)],{ }^{9}$ where $\eta$ is a correcting factor which is related to the reflection losses at the two crystal/air interfaces. $\operatorname{Ps}(\nu, d)$ and $\operatorname{Pr}(\nu, d)$ are the measured transmittances for sample and without sample, respectively. These approximation experiments enabled the quite accurate determination of the power absorption $\alpha(\nu)$ and index of refraction $n(\nu)$ of the sample.

Figures 2(a) and 2(b) give the power absorption spectrum and the refractive index, respectively, for the sample with a thickness of $140 \mu \mathrm{m}$. Some absorption resonance structures in $\alpha(\nu)$ at $0.80 \mathrm{THz}$ 
TABLE I. Assignment of the difference combinations of phonon modes in $\varepsilon$-GaSe.

\begin{tabular}{|c|c|c|c|}
\hline \multicolumn{2}{|c|}{ Wave number $\left(\mathrm{cm}^{-1}\right)$} & $\begin{array}{l}\text { Different combination } \\
\text { assignment }\end{array}$ & $\begin{array}{l}\text { Phonon modes } \\
\left(\mathrm{cm}^{-1}\right)\end{array}$ \\
\hline \multirow{4}{*}{ This study } & \multirow{3}{*}{$\begin{array}{l}\text { Calculated } \\
\text { from modes in } \\
\text { Ref. } 18\end{array}$} & \multirow[b]{3}{*}{$\begin{array}{l}\text { Second-order phonon } \\
\text { modes }\end{array}$} & $A_{1}^{\prime}=307.1$ \\
\hline & & & $A_{2}^{\prime \prime}(\mathrm{LO})=247$ \\
\hline & & & $A_{2}^{\prime \prime}(\mathrm{TO})=236$ \\
\hline & & & $E^{\prime}(\mathrm{LO})=251.5$ \\
\hline 26.7 & 27.3 & $A_{2}^{\prime \prime}(\mathrm{TO})-E^{\prime \prime}$ & $E^{\prime \prime}=208.7$ \\
\hline 41.3 & 42.8 & $E^{\prime}(\mathrm{LO})-E^{\prime \prime}$ & \\
\hline 52.3 & 55.6 & $A_{1}^{\prime}-E^{\prime}(\mathrm{LO})$ & \\
\hline 61.3 & 60.1 & $A_{1}^{\prime}-A_{2}^{\prime \prime}(\mathrm{LO})$ & \\
\hline
\end{tabular}

${ }^{\mathrm{a}}$ Reference 18.

$\left(26.7 \mathrm{~cm}^{-1}\right), \quad 1.24 \mathrm{THz}\left(41.3 \mathrm{~cm}^{-1}\right), \quad 1.57 \mathrm{THz}\left(52.3 \mathrm{~cm}^{-1}\right)$ and $1.84 \mathrm{THz}\left(61.3 \mathrm{~cm}^{-1}\right)$ are attributed to the frequency differences between combinations of acoustical and optical phonons.

The total dielectric function, ${ }^{10-13} \hat{\varepsilon}(\omega)=(n+i \kappa)^{2}$, of a semiconductor consisting of bound electrons, optical phonons, and conduction band electrons in the $\mathrm{THz}$ region approximation is given by

$$
\hat{\varepsilon}(\omega)=\varepsilon(\infty)+\sum_{j=1}^{J} \frac{S_{j} \omega_{\mathrm{TO} j}^{2}}{\omega_{\mathrm{TO} j}^{2}-\omega^{2}-i \Gamma_{j} \omega}-\frac{\omega_{p}^{2}}{\omega\left(\omega+\frac{i}{\langle\tau\rangle}\right)} .
$$

The first term on the right-hand side is from valence electrons, the second term is from polar lattices, and the third term is from conduction electrons/plasmons, where the summation is over the lattice oscillators with the strength $S_{j}$, the resonance transverse optical phonon $\omega_{\mathrm{TO} j}$, the phonon relaxation rate $\Gamma_{j}$, and the average momentum relaxation time for carrier $\langle\tau\rangle$. The mobility is obtained from $\mu=e\langle\tau\rangle / m^{*}$. The valence electron (bound electron) contribution to the high frequency dielectric function is denoted as $\varepsilon_{\infty}$. The static dielectric constant $\varepsilon_{\mathrm{dc}}$ occurs in this treatment as $\varepsilon_{\mathrm{dc}}=\varepsilon_{\infty}$

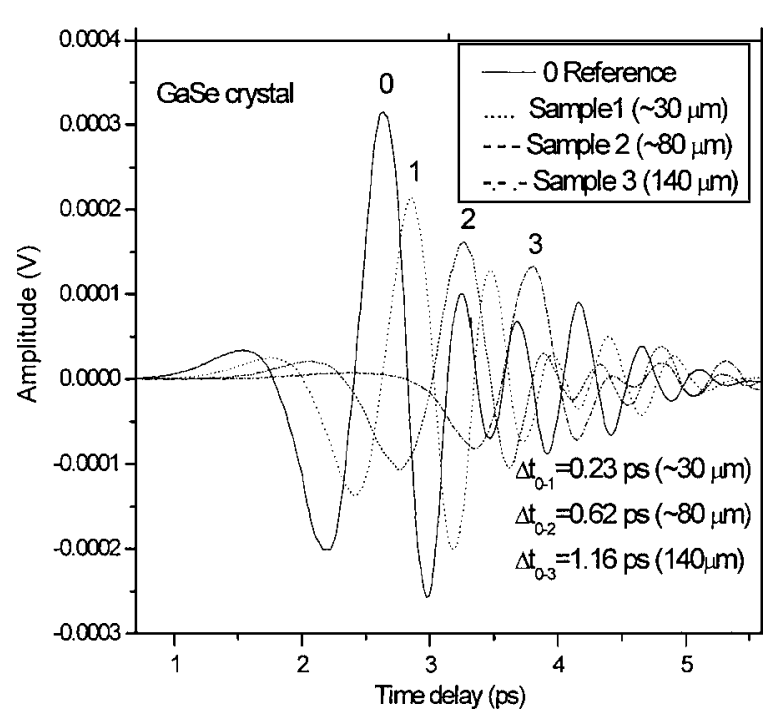

FIG. 1. Time profiles of the THz pulse transmitted through GaSe crystals of different thicknesses.
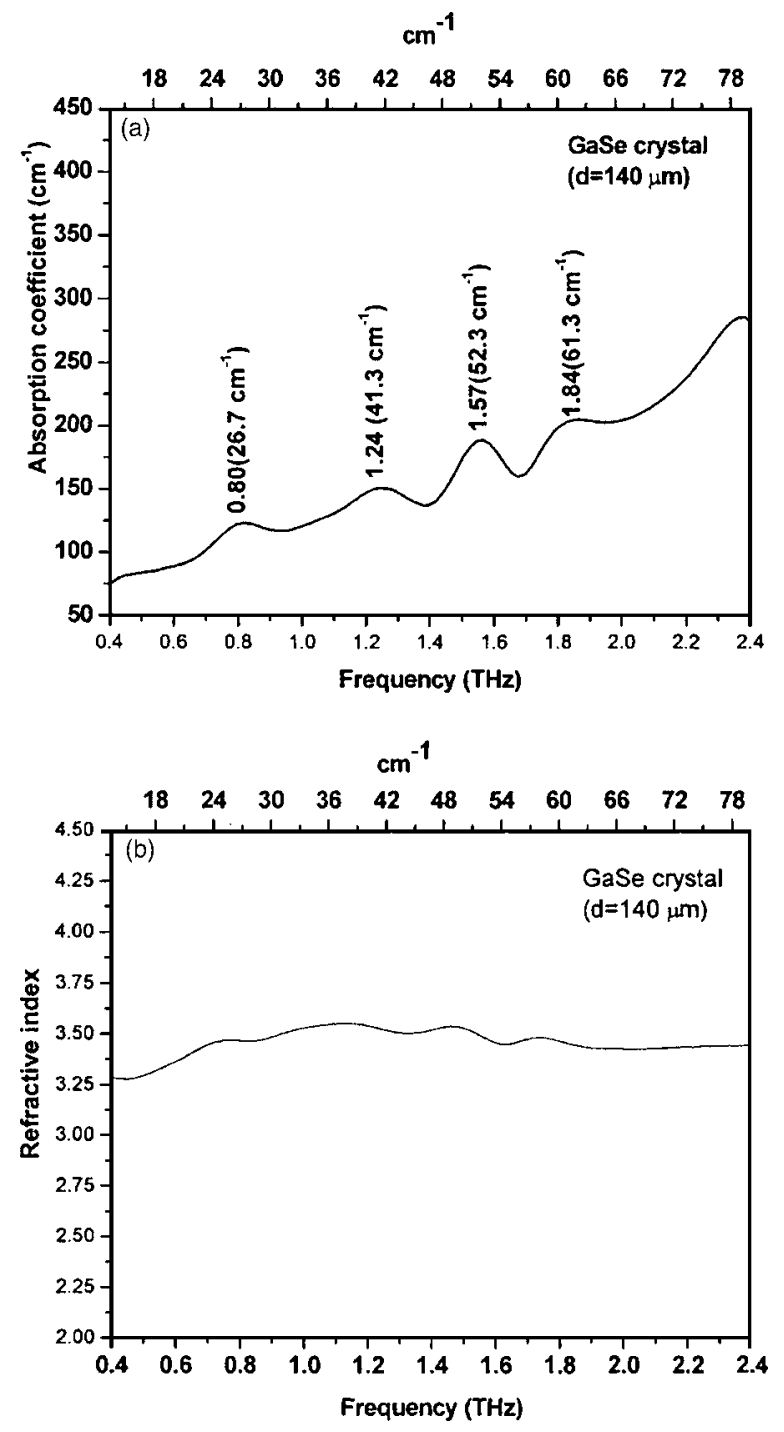

FIG. 2. (a) Power absorption spectrum vs frequency $(\nu)$ of the GaSe crystal $(d=140 \mu \mathrm{m})$. (b) Index of refraction of the GaSe crystal $(d=140 \mu \mathrm{m})$.

$+\sum_{j} S_{j}$. The last term arises from the free carrier contribution where the characteristic plasma frequency $\omega_{p}$ for the free carriers (SI):

$$
\omega_{p}=\sqrt{\frac{e^{2} N}{\varepsilon_{0} m^{*}}},
$$

where $N$ is the free carrier concentration and $m^{*}$ is the electron effective mass. The parameters $\langle\tau\rangle$ and $\omega_{p}$ are obtained from fitting the THz data. The mobility $\mu=e\langle\tau\rangle / m^{*}$ can be found from the momentum relaxation time.

In this study (in the frequency region of $0.4-2.4 \mathrm{THz}$ ), the incident frequency is far away from the TO phonon absorption frequency [see Fig. 2(a)], consequently, Eq. (1) simplifies to the relationship

$$
\begin{aligned}
\hat{\varepsilon}(\omega) & =(n(\omega)+i k(\omega))^{2} \\
& =\varepsilon(\infty)+\frac{\omega_{p}^{2}}{\omega\left(\omega+i\langle\tau\rangle^{-1}\right)}=\varepsilon(\infty)+\frac{i \hat{\sigma}}{\omega \varepsilon_{0}},
\end{aligned}
$$

where $n(\omega)$ and $\kappa(\omega)(=c \alpha / 2 \omega)$ are the real and imaginary parts of the index of refraction, respectively. The complex conductivity is denoted by $\hat{\sigma}(\omega)=\sigma_{r}(\omega)+i \sigma_{i}(\omega)=i \varepsilon_{0} \omega_{p}^{2} /(\omega$ $+i\langle\tau\rangle^{-1}$ ) (Drude model ${ }^{14}$ ) (for $\omega<\omega_{\mathrm{TO}}$ ), where $\varepsilon_{0}(=8.854$ 

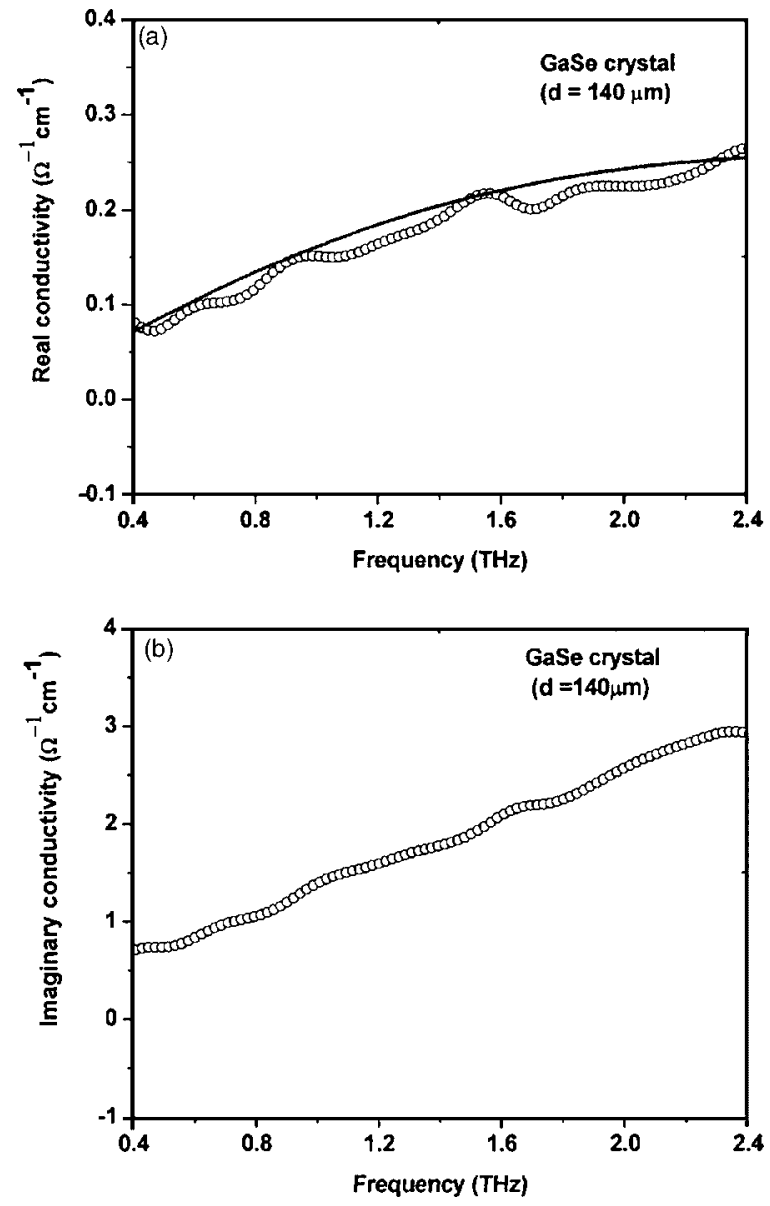

FIG. 3. Complex conductivity for the real part $\sigma_{r}(\nu)$ and the imaginary part $\sigma_{i}(\nu)$ in the THz region. The solid line in (a) is fitted by the equation $\sigma_{r}$ $=\left(\varepsilon_{0} \omega_{p}^{2}\langle\tau\rangle^{-1}\right) /\left(\omega^{2}+\langle\tau\rangle^{-2}\right)$ using the parameters $\langle\tau\rangle=56 \mathrm{fs}$ and $\omega_{p}=2.6 \mathrm{THz}$.

$\left.\times 10^{-12} \mathrm{~F} / \mathrm{m}\right)(\mathrm{MKS})$ is the free-space permittivity. Substituting $\hat{\sigma}(\omega)=\sigma_{r}(\omega)+i \sigma_{i}(\omega)$ in the right-hand side of Eq. (3), the real and imaginary parts of conductivity $\hat{\sigma}(\omega)$ are given by

$$
\sigma_{i}(\omega)=\varepsilon_{0} \omega\left(\varepsilon(\infty)-n^{2}+\kappa^{2}\right),
$$

and

$$
\sigma_{r}(\omega)=2 n \kappa \omega \varepsilon_{0},
$$

where $\varepsilon(\infty)=7.443$ is used. ${ }^{15}$ Equations (4) and (5) in conjunction with the experimental data from the of $\alpha(\nu)$ and $n(\nu)$ curves shown in Figs. 2(a) and 2(b), the real and imaginary parts of the conductivity were calculated and are shown in Figs. 3(a) and 3(b).

Resonance structures at the four prominent resonance peaks in $\alpha(\nu)$ are observed at $0.80 \mathrm{THz}\left(26.7 \mathrm{~cm}^{-1}\right)$, $1.24 \mathrm{THz}\left(41.3 \mathrm{~cm}^{-1}\right), \quad 1.57 \mathrm{THz}\left(52.3 \mathrm{~cm}^{-1}\right), \quad$ and $1.84 \mathrm{THz}\left(61.3 \mathrm{~cm}^{-1}\right)$. These peaks are attributed to frequency difference combinations of second order acoustical and optical phonons such as LO-TA and other mode combinations (see Table I) ${ }^{16,17}$ The band at $0.8 \mathrm{THz}$ is assigned to $A_{2}^{\prime \prime}(T O)-E^{\prime \prime}\left(27.3 \mathrm{~cm}^{-1}\right)$. According to Raman and infrared measurements ${ }^{17}$ of $\varepsilon$-type GaSe the band at $1.24 \mathrm{THz}$ is from the $E^{\prime}(\mathrm{LO})-E^{\prime \prime}\left(42.8 \mathrm{~cm}^{-1}\right)$ difference mode. The $1.57 \mathrm{THz}$ feature could be explained as an $A_{1}^{\prime}-E^{\prime}(\mathrm{LO})\left(55.6 \mathrm{~cm}^{-1}\right)$ process. The absorption at $1.84 \mathrm{THz}$ was assigned to $A_{1}^{\prime}-A_{2}^{\prime \prime}(\mathrm{LO})\left(60.1 \mathrm{~cm}^{-1}\right)$, which was also observed in Raman measurements.
From the relationship of $\hat{\sigma}(\omega)=\sigma_{r}(\omega)+i \sigma_{i}(\omega)$ $=i \varepsilon_{0} \omega_{p}^{2} /\left(\omega+i\langle\tau\rangle^{-1}\right)$, the real and imaginary parts of $\hat{\sigma}(\omega)$ are given by

$$
\sigma_{r}(\omega)=\frac{\varepsilon_{0} \omega_{p}^{2}\langle\tau\rangle^{-1}}{\omega^{2}+\langle\tau\rangle^{-2}}
$$

and

$$
\sigma_{i}(\omega)=\frac{\varepsilon_{0} \omega \omega_{p}^{2}}{\omega^{2}+\langle\tau\rangle^{-2}} .
$$

The fit to the measured absorption coefficient $\alpha(\nu)$ and the index of refraction $n(\nu)$ is quite good over the entire frequency range, as is the fit to the real part of $\sigma_{r}(\omega)$ [see Fig. 3(a)]. Two parameters were used to fit the experimental data $\sigma_{r}(\omega)$ [real part of $\left.\sigma(\omega)\right]$; the plasma frequency $\omega_{p}$ $=2.6 \pm 0.2 \mathrm{THz}$ and the momentum relaxation time $\langle\tau\rangle$ $=56 \pm 2$ fs. Using the effective mass for $m^{*}=0.011 m_{0}$ (Ref. 19) $\left(m_{0}=9.1 \times 10^{-31} \mathrm{~kg}\right)$, where $m_{0}$ is the free-electron mass, the carrier density $N=1.0 \times 10^{15} / \mathrm{cm}^{3}$, and the electron mobility $\mu=89 \mathrm{~cm}^{2} / \mathrm{V}$ s were obtained. This mobility value extracted from $\mathrm{THz}$ data is in close to the reported value of $75 \mathrm{~cm}^{2} / \mathrm{V} \mathrm{s}$ (Ref. 20) and the time of flight mobility of $102 \mathrm{~cm}^{2} / \mathrm{V} \mathrm{s}$ measured on a sample grown from the same batch of the GaSe crystal. ${ }^{21}$

The key parameters of the momentum relaxation time, $56 \mathrm{fs}$, the mobility, $89 \mathrm{~cm}^{2} / \mathrm{V} \mathrm{s}$, and the carrier density, $10^{15} / \mathrm{cm}^{3}$, were obtained from the $\mathrm{THz}$ dielectric response of a GaSe crystal from 0.4 to $2.4 \mathrm{THz}$. Second order acoustical and optical phonon resonances are observed in the absorption spectrum in the region from 0.4 to $2.4 \mathrm{THz}$.

This work is supported in part by NASA, DoD Center, NYSTAR, and CUNY Organized Research. The authors thank Dr. Hakan Altan for helpful discussions.

${ }^{1}$ R. Huber, A. Brodschelm, F. Tauser, and A. Leitenstorfer, Appl. Phys. Lett. 76, 3191 (2000)

${ }^{2}$ K. Liu, J. Xu, and X.-C. Zhang, Appl. Phys. Lett. 85, 863 (2004).

${ }^{3}$ N. Piccioli, R. Le Toullec, M. Mejatty, and M. Balkanski, Appl. Opt. 16, 1236 (1976).

${ }^{4}$ W. Shi, Y. Ding, and K. Vodopyanov, Opt. Lett. 27, 1454 (2002).

${ }^{5}$ T. Jeon, D. Grischkowsky, A. Muherjee, and R. Menon, Appl. Phys. Lett. 77, 2452 (2000).

${ }^{6}$ E. Pickwell, B. Cole, A. Fitzgerald, V. Wallace, and M. Pepper, Appl. Phys. Lett. 84, 2190 (2004).

${ }^{7}$ B. Yu, F. Zeng, Y. Yang, Q. Xing, A. Chechin, X. Xin, I. Zeylikovich, and R. R. Alfano, Biophys. J. 86, 1649 (2004).

${ }^{8}$ M. Schall, M. Walther, and P. Uhd Jepsen, Phys. Rev. B 64, 094301 (2001).

${ }^{9}$ B. L. Yu, F. Zeng, Q. Xing, and R. R. Alfano, Appl. Phys. Lett. 82, 4633 (2003).

${ }^{10}$ R. Tsu, Phys. Rev. 164, 380 (1967).

${ }^{11}$ M. Born and K. Huang, The Dynamical Theory of Crystal Lattice (Clarendon, Oxford, 1956).

${ }^{12}$ R. R. Alfano, Phys. Lett. 29A, 634 (1969).

${ }^{13}$ K. Moller and W. Rothschild, Far-Infrared Spectroscopy (Wiley, New York, 1971), Chap. 12.

${ }^{14}$ M. Beard, G. Turner, and C. Schmuttenmaer, Phys. Rev. B 62, 15764 (2000).

${ }^{15}$ N. Piccioli, R. Toullec, M. Mejatty, and M. Balkanski, Appl. Opt. 16, 1236 (1977)

${ }^{16}$ M. Hayek, O. Brafman, and R. M. A. Leith, Phys. Rev. B 8, 2772 (1973).

${ }^{17}$ H. Yoshida, S. Nakashima, and A. Mitsuishi, Phys. Status Solidi B 59, 655 (1973)

${ }^{18}$ N. Kuroda, O. Ueno, and Y. Nishina, Phys. Rev. B 35, 3860 (1987).

${ }^{19}$ D. M. Todorovic and P. M. Nikolic, Appl. Opt. 24, 2252 (1985).

${ }^{20} \mathrm{httn}: / /$ www rmdinc.com/semiconductor.PDF: data compiled by RMD Inc

${ }^{2 \mathrm{i}}$ EIC Laboratories (private communication). 